\title{
Gentrificación, movilidad y transporte: aproximaciones conceptuales y ejes de indagación ${ }^{1}$
}

\author{
Jorge Blanco ${ }^{2}$, Luciana Bosoer ${ }^{3}$ y Ricardo Apaolaza ${ }^{4}$
}

\begin{abstract}
RESUMEN
El presente artículo tiene como objetivo plantear las bases para analizar las relaciones entre los procesos de gentrificación, el transporte y la movilidad, a partir de la identificación de un limitado desarrollo de esta problemática, particularmente en América Latina. Luego del desarrollo de los conceptos que organizan el campo de indagación (gentrificación, transporte, movilidad, accesibilidad, capital espacial), se presentan tres ejes organizadores. El primero sugiere analizar el papel de las inversiones en infraestructura y servicios de transporte como posible dispositivo gentrificador. El segundo pone el foco en la atracción diferencial de la accesibilidad en el despliegue de procesos de gentrificación, en distintos contextos territoriales. El tercero propone analizar los efectos sobre la movilidad cotidiana de los grupos desplazados por los procesos de gentrificación. Estos ejes abren una agenda de investigación que avanza sobre la complejidad de la gentrificación, incorporando nuevas dimensiones.
\end{abstract}

Palabras clave: Gentrificación, movilidad, transporte, capital espacial.

\begin{abstract}
This paper aims to lay the groundwork for analysis of the relationships between the processes of gentrification, transport and mobility, by identifying the limited development of this field, particularly in Latin America. After the development of the concepts that organize the field of inquiry (gentrification, transport, mobility, accessibility, spatial capital), the article presents three axes of organization. The first one attempts to analyze the role of investment in transport infrastructure and services as possible mechanisms of gentrification. The second focuses on the differential attraction to accessibility in the deployment of gentrification in different territorial contexts. The last axis seeks to examine the effects on daily mobility of the groups displaced by gentrification. These axes open a research agenda that advances on the complexity of gentrification, adding new dimensions.
\end{abstract}

Key words: Gentrification, mobility, transport, spatial capital.

\footnotetext{
1 La elaboración de este texto se desarrolla en el marco del proyecto de investigación "CONTESTED_CITIES - Contested Spatialities of Urban Neoliberalism: Dialogues between Emerging Spaces of Citizenship in Europe and Latin America", financiado por la Comisión Europea (Grant Agreement: PIRSES-GA-2012-318944). Artículo recibido el 10 de octubre de 2013, aceptado el 2 de marzo de 2014 y corregido el 20 de mayo de 2014.
}

\footnotetext{
2 Instituto de Geografía, Universidad de Buenos Aires (Argentina). E-mail: jblancodis@gmail.com

3 Instituto de Geografía, Universidad de Buenos Aires (Argentina). E-mail: lucianabosoer@gmail.com

4 Instituto de Geografía, Universidad de Buenos Aires (Argentina). E-mail: ricardoapaolaza@yahoo.com.ar
} 
Los estudios sobre los procesos de gentrificación desarrollados en el marco de las políticas urbanas neoliberales abarcan una amplia gama temática, tanto en la literatura producida en ámbitos anglosajones, como en la desarrollada en España y América Latina. Estos estudios presentan algunos puntos en común, pero también importantes especificidades (Janoschka et al., 2013). En el caso latinoamericano, estos autores dan cuenta del carácter emergente de la temática y de la utilización preferencial de términos tales como renovación, revitalización y rehabilitación para referirse a las estrategias de planificación urbana desarrolladas en las últimas décadas. De alguna manera, estos términos han actuado como sinónimos de la gentrificación, articulando una amplia temática que gira alrededor de la gentrificación simbólica, las políticas neoliberales urbanas que pueden desencadenar procesos de gentrificación (con énfasis en los procesos liderados por el Estado), la conformación de nuevos mercados inmobiliarios en áreas centrales y periféricas, y los movimientos de resistencia a las políticas neoliberales (Janoschka et al., 2013).

Esta amplitud de debates habilita a incorporar nuevos aspectos que apunten, por un lado, a hacer aún más compleja la mirada sobre las condiciones de producción, apropiación y uso diferencial de la ciudad y, por otro, que den cuenta de algunos de los efectos diferenciados que los procesos de gentrificación tienen en la vida cotidiana. En este sentido se propone incorporar las desigualdades en el acceso a las condiciones de transporte y movilidad como una dimensión significativa para el análisis de los procesos de gentrificación.

Conviene explicitar dos puntos de partida que enmarcan esta propuesta. En primer lugar, extendemos la noción de gentrificación a una situación en la que se presentan los siguientes rasgos (Rérat \& Lees, 2011; Casgrain y Janoschka, 2013):

1. la reinversión de capital en un espacio definido y un alza correspondiente del valor del suelo de ese espacio o en áreas colindantes;

2. Ia llegada de agentes con mayor capacidad de pago que los usuarios establecidos en ese espacio o en áreas colindantes;
3. cambios en las actividades y en el paisaje urbano controlados por los grupos que ingresan al territorio en cuestión;

4. el desplazamiento directo, o la presión indirecta para el desplazamiento, de grupos sociales de ingresos más bajos de los que entran.

En segundo lugar, proponemos considerar la doble dimensión de fijación y movilidad (Cresswell, 2006; Rérat \& Lees, 2011) representada, en el análisis de la gentrificación, por la fijación de residencia o del lugar de actividad en un universo relacional accesible a través de la movilidad. De esta manera se recalifican los lugares en proceso de gentrificación no solo en función de sus propias condiciones sino en relación con las nuevas coordenadas urbanas, claves para la definición de las necesidades de desplazamiento. Se retoma así un tema trabajado en profundidad en la geografía latinoamericana, como es el interés por los "fijos" (la construcción material fijada) y los flujos (las interacciones y el movimiento) (Santos, 1996). La combinación de fijación y movilidad también abre la posibilidad de extender la apropiación urbana diferencial implícita en los procesos de gentrificación, al uso temporal del territorio sin fijación.

A partir de estas premisas, el presente trabajo tiene como objetivos centrales explorar en términos conceptuales las relaciones entre gentrificación, transporte y movilidad, y proponer una serie de ejes de indagación que orienten las investigaciones en este campo. Asimismo, se espera que esta primera instancia de indagación conceptual aporte a los debates generados en el marco de la Red Contested Cites- Ciudades en Disputa y permita la posterior selección y desarrollo de estudios de casos

\section{Una trama conceptual para abordar el problema}

Tres aproximaciones conceptuales nos proveen un entramado apropiado para analizar las relaciones entre los procesos de gentrificación y la movilidad cotidiana. En primer lugar, el campo de indagación en torno a la problemática del transporte, de fuerte presencia en los estudios sobre la estructuración 
y los cambios urbanos. En segundo lugar, el concepto de movilidad, que refleja un paradigma emergente en el análisis de las sociedades contemporáneas. En tercer lugar, los conceptos de capital espacial y de motility, que resumen coordenadas socioterritoriales en el análisis de la movilidad.

La perspectiva basada en el concepto de transporte ha sido la estructuradora central de las investigaciones en la materia. Como campo de investigación, contiene un amplio espectro de preocupaciones en torno a la organización de las redes y los sistemas, las infraestructuras y los servicios, que facilitan los desplazamientos de bienes y personas, en distintas escalas en el territorio y a través de diferentes modos de transporte. Los sistemas de transporte son "herramientas para el dominio del espacio y respuestas al obstáculo de la distancia, son creadores de continuidades al permitir las conexiones, los contactos, las complementariedades y las interacciones sin las cuales ningún sistema socioespacial podría funcionar" (Bavoux et al., 2005: 5). La organización y las características de los sistemas de transporte tienen implicancias críticas en la conformación y dinámica del espacio urbano, al distribuir diferencialmente atributos de accesibilidad y valorización entre los distintos lugares de la ciudad (Miralles, 2002; Blanco, 2010).

Desde fines de la década de 1990, pero particularmente desde el año 2000, ha cobrado importancia un enfoque diferente para este campo problemático, que se basa en el concepto de movilidad (Gutiérrez, 2009). La movilidad, concebida como una necesidad y como un derecho (Ascher, 2004), da cuenta de las posibilidades de acceso a los servicios básicos para el desarrollo de la vida social, para la participación activa en términos económicos, y para la sociabilización de las personas. Cada vez más, las posibilidades de movilidad cualifican a personas y lugares y están incorporadas sistemáticamente en conceptos tales como derecho a la ciudad, vivienda apropiada y desarrollo sostenible, proponiéndose incluso "el paradigma de la movilidad" (Urry, 2007) como una lente que permite analizar las sociedades contemporáneas.

La movilidad toma como punto de partida las necesidades de los sujetos y grupos socia- les y pone en juego un conjunto de "relaciones sociales" (Levy, 2001) y de "prácticas sociales" (Gutiérrez, 2009), vinculadas con los procesos de producción y de reproducción social, espacial y temporalmente definidos, que se manifiestan en la construcción y el uso del territorio. Este uso articula necesidades sociales, representaciones del espacio y localizaciones de los lugares, redes y medios materiales, tiempos y estrategias de los sujetos, para la consecución de los desplazamientos (Blanco et al., 2013). Es decir, cruza de manera crítica el conjunto de actividades propias del desarrollo de la vida social con su articulación en el espacio y en el tiempo.

En términos más generales, la movilidad aparece íntimamente ligada con el derecho a la ciudad y con el capital social de las personas (Levy, 2001; Orfeuil, 2003; Cebollada, 2006 y 2009; Díaz Olivera et al., 2008; AveIlaneda y Lazo, 2011). De esta manera, antes que un atributo espacial definido a partir de un sistema de transporte, se pone el acento en la diferenciación social, etaria, de género, implícitos en el concepto de movilidad.

Tanto el transporte como la movilidad pueden asociarse con la accesibilidad, que presenta dos interpretaciones principales. Por un lado, se ha considerado a la accesibilidad como una cualidad de los lugares, que remite a la facilidad para alcanzar desde cada uno de esos lugares un conjunto de puntos significativos del territorio (en cualquiera de las escalas). De esta manera, la accesibilidad coloca cada lugar en un universo relacional, de posiciones relativas, que se traduce en una ampliación de las posibilidades de movilidad para el desarrollo de la vida cotidiana. Por otro, en las últimas dos décadas, y en el marco de la generalización de la perspectiva de la movilidad, va cobrando cada vez más importancia la consideración de la accesibilidad, más precisamente de las posibilidades de acceso, como una cualidad de los individuos y de los grupos sociales (Levy, 2001). Esta perspectiva abre decididamente la puerta para el análisis de una diferenciación social de la accesibilidad, aun para quienes residen en un mismo lugar, e incluso disponen de similares niveles de ingreso o de instrucción (Jouffe, 2011). Entendemos que, como herramientas de indagación, ambas interpretaciones son de gran utilidad para reconstruir 
condiciones estructurales y subjetivas de la movilidad.

Un concepto articulador de esta entrada a las necesidades y los problemas de desplazamiento en la ciudad es el de "motilidad" o motility (Kaufmann et al., 2004), expresado también como "capital espacial" (Rérat \& Lees, 2011), tomando como punto de partida las propuestas de Pierre Bourdieu. Motility es el capital espacial del que disponen los individuos o los hogares, es la capacidad "de ser móviles en el espacio social y geográfico" y los modos en que "acceden y se apropian de la movilidad socioespacial de acuerdo con sus circunstancias" (Kaufmann et al., 2004: 750). La motility requiere de recursos y habilidades, relacionadas con el funcionamiento de los hogares, con las capacidades para tramitar la flexibilidad, la adaptación y las incompatibilidades en el tiempo y en el espacio. En sentido amplio, se combinan modos de transporte y diferentes formas de movilidad (física, virtual, servicio de mensajes cortos, sms, teléfono, etcétera). En este marco, es central la hipótesis de que estas capacidades de movilidad no constituyen únicamente un capital individual o una habilidad innata sino que dependen de la estructura de las familias y de cómo funcionan.

El capital espacial comprende tres componentes: acceso, competencia y apropiación (Kaufmann et al., 2004). El acceso, está relacionado "con el abanico de movilidades posibles de acuerdo con el lugar, el tiempo y otras restricciones contextuales" (Rérat \& Lees, 2011: 127). Entre estas restricciones, la disponibilidad económica cobra una particular importancia. El acceso tiene una estrecha relación con la oferta de redes de todo tipo (viales, de servicios públicos, peatonales, de senderos seguros, etcétera) que canalizan la circulación uniendo puntos significativos de la ciudad, con una cierta cobertura temporal.

La competencia, por su parte, remite a "las habilidades de los individuos" (Rérat \&Lees, 2011: 127). Es decir, a un conjunto de capacidades derivadas de cuestiones físicas (edad, capacidades diferenciales), adquiridas (disponibilidad de registro para conducir, conocimiento de las redes y de los dispositivos de pago y de combinaciones entre modos de transporte) y organizacionales (planificación, lecturas de la señalización y las indicaciones para el uso de los modos y sus combinaciones, etcétera).

El tercer atributo del capital espacial es la apropiación, que "se refiere a las estrategias, motivaciones, valores y prácticas de los individuos" (Rérat \& Lees, 2011: 127). Esta apropiación remite a las dimensiones del espacio percibido, representado y usado a través de las prácticas. Para la apropiación efectiva resulta de gran importancia la representación social que tienen los modos de transporte (por ejemplo la idea de libertad que brinda el automóvil particular, las implicancias que puede tener el compartir con otros el transporte público, las rutas fijas y los cronogramas establecidos o la flexibilidad de horarios y recorridos, la sociabilidad del espacio público, etcétera).

El contexto urbano y el contexto de los hogares en los que se despliegan estos tres componentes (acceso, competencia y apropiación) son críticos. Así como los hogares plantean una serie de necesidades de movilidades diferenciales en función de sus niveles de ingreso, composición, edades y géneros; los contextos urbanos plantean coordenadas espaciales concretas de materialización de las redes y las localizaciones que dan "forma urbana" a las necesidades específicas de movilidad.

En un aporte pionero, Françoise Dureau (1999) propuso una mirada integrada sobre residencia y movilidad, fijación y flujos, en distintas escalas temporales y espaciales, que puede ser de utilidad para analizar los procesos de gentrificación. Esta autora pone el acento en la comprensión del "espacio residencial de los individuos, configuración espacial definida por el conjunto de los lugares movilizados (frecuentados o no) en un momento dado por un individuo, y el sistema residencial, noción vecina a la anterior pero que insiste en la articulación entre los lugares y en las prácticas que los ponen en relación. Estas nociones vuelven a considerar el espacio "potencialmente accesible" (en función del sistema de transporte) a partir de una localización residencial y el espacio movilizado, practicado en un momento dado, por los individuos y los hogares en sus diferentes actividades" (Dureau, 1999: 333). 
Esta perspectiva implica considerar a la vivienda, la fijación residencial, no solo en función de sus características físicas sino en términos de su accesibilidad a los lugares de empleo, los grandes equipamientos, las áreas comerciales y de servicios, las zonas recreativas; situarla, en consecuencia, en un universo relacional en el seno de la estructura urbana. Desde el punto de vista de la gentrificación, estas nociones permiten recuperar lo que implica cada localización en términos de relación potencial con otras localizaciones y ponerla en movimiento en el tiempo, siguiendo los cambios en el espacio residencial de los individuos a través de las trayectorias residenciales y cotidianas.

Por último, proponemos sostener un alerta metodológico que coloque en primer plano las articulaciones y tensiones que se generan en los procesos de constitución de los lugares y en la apropiación y los usos del territorio (Massey, 2000; Blanco, 2010). Si las articulaciones dan cuenta de las dinámicas asociadas, las tensiones Ilaman la atención sobre los conflictos manifiestos y potenciales. En relación con el objetivo del artículo, cobra singular relevancia la contrastación simultánea de la movilidad (o hipermovilidad) y de la fijación (Massey, 2000) como "dos caras de la misma moneda" (Rérat \& Lees, 2011: 127), fuente de tensiones y conflictos.

A pesar de la importancia creciente de la movilidad en la agenda de la investigación social, aún no hay un gran desarrollo de estudios sobre las relaciones entre gentrificación, transporte y movilidad. En alguno de los pocos antecedentes sobre el tema se señala explícitamente esta carencia, al sostener que "desde mediados de la década de 1980, la gentrificación ha sido analizada, principalmente, en términos de la nueva clase media que marca su distinción social de las masas suburbanas; en comparación, los análisis de las ventajas locacionales y las prácticas de movilidad han prácticamente desaparecido" (Rérat \& Lees, 2011: 126). En consecuencia, se presenta como un campo de interés para indagar sobre aspectos específicos de las políticas urbanas neoliberales.

Para (re)insertar la movilidad en el estudio de la gentrificación, proponemos organizar algunas ideas que ayuden a explorar esta re- lación a partir de la identificación de tres ejes articuladores. El primero de ellos vincula la gentrificación con la infraestructura de circulación y los servicios de transporte; el segundo eje indaga en el juego de la accesibilidad diferencial como componente de los procesos de gentrificación; y el tercero se enfoca en el movimiento del espacio de movilidad cotidiana de los grupos sociales desplazados por los procesos de gentrificación.

\section{Tres ejes para indagar en la relación gentrificación, transporte y movilidad}

\author{
El papel de la infraestructura y los \\ servicios de transporte como un \\ dispositivo gentrificador
}

La inversión en infraestructura y servicios de transporte promueve modificaciones en la accesibilidad y en las posiciones relativas de un lugar en el marco general de la ciudad. Con frecuencia ha sido una pieza clave en materia de intervenciones urbanas, actuando como disparador de procesos de renovación y de recualificación, concebidos bajo el paradigma de los "efectos estructurantes" del transporte en relación con el espacio urbano (Offner, 1993).

En ocasiones, han sido las propias infraestructuras de transporte las que han sido objeto de los procesos de reestructuración urbana, como las refuncionalizaciones portuarias y de los water fronts, de los que existen ejemplos tanto en América Latina (Guayaquil, Buenos Aires, Rosario, Río de Janeiro) como en diversos lugares del mundo (Toronto, Londres, Hamburgo, Barcelona, entre otros) (Cuenya y Corral, 2011; Desfor et al., 2011). En otras ocasiones, se trata de la incorporación de innovaciones tecnológicas (nuevas líneas, nuevos vehículos, nuevas condiciones de circulación, vías segregadas), que crean nuevos entornos residenciales y cambian las condiciones de acceso a lugares de trabajo, compras y recreación.

El análisis de los impactos de estas innovaciones en la infraestructura y en los sistemas de circulación forma parte de las evaluaciones habituales para este tipo de obras, tanto en sus versiones académicas como en las 
implicadas directamente en la gestión del territorio. En particular las evaluaciones hacen foco en dos tipos de efectos. Por un lado, los vinculados directamente con la circulación, como la reducción de los tiempos de viaje, la mejora en la conectividad, la posibilidad de mejorar la distribución modal atrayendo usuarios del automóvil particular, etcétera. Por otro, se analizan los efectos relacionados con la estructura y dinámica urbana, como el aumento de los precios de los inmuebles ubicados en el área de influencia, los cambios o la intensificación de usos del suelo o el desarrollo de nuevas centralidades comerciales o de servicios sobre las trazas de los servicios de transporte o en el entorno de las estaciones. Dos ejemplos recientes de América Latina, en los que se evalúan los efectos de nuevos sistemas de buses rápidos (BRT) sobre los precios del suelo, son los presentados por Rodríguez y Mojica (2008) y por Verlandia (2013), sobre Bogotá y Ciudad de México, respectivamente.

Menor ha sido la atención sobre los efectos de estas innovaciones en materia de transporte sobre los distintos grupos sociales y las movilidades residenciales, tanto en lo que se refiere a la llegada de nuevos residentes como a los desplazamientos que se producen por la nueva oferta de transporte. Algunos estudios desarrollados en Estados Unidos y Canadá (Pollack et al., 2010; Grube-Cavers y Patterson, 2013) han alertado sobre el efecto gentrificador que puede aparecer asociado a la expansión de la oferta de transporte público urbano basado en medios guiados, arrojando evidencia cuantitativa preliminar sobre la correlación e intensidad entre ambos fenómenos. Se ha destacado, asimismo, la gran dificultad metodológico-operativa asociada a la búsqueda de patrones de correlación entre dos fenómenos de gran complejidad como son la movilidad y la gentrificación (GrubeCavers \& Patterson, 2013).

En relación con esta línea, un aspecto central de las mencionadas innovaciones es la carga simbólica que conllevan los procesos de modernización, en particular cuando se los asocia con el paradigma de la movilidad sostenible, como sucede con la ampliación de los modos guiados, las vías segregadas y todo aquello que implique modelos más eficientes de consumo energético o control de emisiones (metros, tranvías, sistemas BRT, ci- clovías y áreas peatonalizadas, etcétera). Esta construcción de una imagen moderna y sostenible, apoyada en los servicios de transporte, puede ser un componente importante para el despliegue de los procesos de gentrificación.

En todos los casos, el papel estatal es clave en la definición, construcción y habilitación de estas infraestructuras y de los servicios de transporte. Por ello es que, desde el punto de vista de la gentrificación, estas acciones podrían encuadrarse bajo el rótulo del "liderazgo estatal" (state led), con una "alta inversión de capital en un espacio definido y un alza correspondiente del valor del suelo en ese espacio y en los espacios colindantes" (Casgrain y Janoschka, 2013). Este liderazgo estatal en materia de transporte no obsta que se haya desplegado un amplio abanico de políticas neoliberales en las últimas décadas, con notorios procesos de privatizaciones, concesiones y asociaciones público-privadas, implementados en una gran cantidad de países (CEPAL, 2005).

Las iniciativas en materia de mejora de los sistemas de transporte son percibidas como de gran necesidad, en particular en las grandes metrópolis latinoamericanas en las que la movilidad cotidiana aún representa un grave problema para amplias mayorías de la población, pero al mismo tiempo generan fuertes tensiones al asociarse esas mejoras con la complejidad de los procesos urbanos y con efectos de mayor segregación, fragmentación y diferenciación social. Figueroa da cuenta de algunos de estos problemas en el caso de las ciudades latinoamericanas, al afirmar que "la polarización social ha tenido sus expresiones tanto en la oferta como en la demanda de transporte: desde el punto de vista de la demanda, el servicio se ha segmentado para consagrar las crecientes desigualdades económicas que se expresan también en el acceso diferenciado al servicio. Desde el punto de vista de la oferta, se ha visto cómo surge una nueva actividad de transporte, más informal y precaria, que da cuenta de la flexibilización de los mercados de trabajo y de las oportunidades que ofrece para insertarse en las tendencias de la tercerización del empleo, mientras también se consolidan variadas formas de movilidad de los sectores de mayores ingresos, desde el automóvil hasta los servicios selectivos y exclusivos de transporte público" (Figueroa, 2005: 52). 
En este marco cabe preguntarse si es inevitable que la implantación de nuevos sistemas de circulación o que una renovación de los existentes se traduzca en un dispositivo gentrificador (Sequera, 2013), y derive ineludiblemente en una apropiación por parte de ciertos grupos sociales de esas mejores condiciones de circulación. Vale aquí una pregunta complementaria: ¿Qué conjunto de políticas pueden traducirse en mejoras en la accesibilidad y en las condiciones de movilidad de la población sin que formen parte de procesos de gentrificación? Pollack et al. (2010) brindan algunas sugerencias básicas, al proponer herramientas para desarrollar políticas integradas en torno a la planificación urbana, a la regulación del mercado inmobiliario y al manejo del transporte y la movilidad. Creemos que se trata de tres aspectos claves, en particular el referido al mercado inmobiliario ya que es a través de la fijación residencial y de los desplazamientos de población con residencia previa en el lugar, como se manifiestan de manera dominante los procesos de gentrificación.

Un caso que requiere particular atención y seguimiento es el de Medellín, donde se produjeron mejoras significativas en la movilidad a través de inversiones en transporte público ("metrocable") dirigidas a los sectores pobres de la ciudad, enmarcadas en un conjunto de intervenciones urbanas. Se han identificado cambios importantes manifiestos en cierta renovación comercial, mejoras en las viviendas y la inclusión de los barrios servidos por el metrocable entre los lugares turísticos de la ciudad, tanto para los residentes en la propia ciudad como para los turistas del resto del país y del exterior (Leibler y Brand, 2012). La aplicación de políticas integrales dificulta la asignación directa de la inversión en transporte como causa en la generación de cambios sociales y económicos, pero recupera la complejidad de la dinámica urbana desafiando las perspectivas mecanicistas y abogando por más políticas territoriales integradas (Blanco, 2010; Dávila y Daste, 2011).

\section{La accesibilidad diferencial como atractivo para la gentrificación}

El foco de este segundo eje está dirigido a evaluar el papel que juegan las condiciones de accesibilidad en los procesos de gentrificación. Tal como fue señalado, la accesibili- dad tiene dos dimensiones: una refiere a los lugares y otra a los grupos sociales. Vistas en articulación, se requiere una mirada socioterritorial: son los diferentes grupos sociales "localizados" en contextos territoriales los que se apropian o toman ventaja de las condiciones de accesibilidad diferencial de los lugares. Por un lado, las condiciones materiales de producción del espacio urbano definen características particulares de diferenciación del conjunto de actividades y usos del suelo (localizaciones, diversidad y combinación, intensidades y densidades variadas) y de las distintas redes urbanas. Por el otro lado, los sistemas de actividades cotidianas están en estrecha relación con las condiciones de reproducción de los hogares, con sus necesidades y posibilidades particulares.

En consecuencia, para evaluar la accesibilidad diferencial es necesario identificar los diferentes contextos territoriales y los perfiles socioeconómicos de los hogares. Una amplia variedad de casos de gentrificación se produce en antiguos barrios con un alto nivel de consolidación en las inmediaciones de las áreas centrales. Estos barrios, en general, cuentan con buenas condiciones de accesibilidad; ofrecen cercanía al centro metropolitano, fácil acceso a lugares de empleos, de compras y de recreación; oferta diversificada de transporte público, y posibilidad de desplegar trayectos cortos, es decir, articular entornos residenciales con las actividades de la vida cotidiana en un esquema de proximidad.

Rérat y Lees sostienen que, además de la proximidad, es importante el rápido acceso a redes más amplias, "porque una de las razones por las cuales los gentrificadores eligen vivir en las áreas centrales es para incrementar su capital espacial" (2011: 128). Estas localizaciones centrales contribuyen a combinar trabajo, vida social y familiar para cierto perfil de los hogares, a través de la reducción de los tiempos de viaje y la posibilidad de inserción relativamente rápida en las redes de circulación más importantes, que permiten multiplicar la llegada aún a lugares lejanos.

En uno de los pocos casos de estudio sobre ciudades latinoamericanas, investigaciones empíricas dan cuenta del atractivo de barrios pericentrales de Santiago de Chile desde el punto de la movilidad, tanto en la escala 
local como en la metropolitana y regional: "Lo nuevo de estos móviles urbanos es que sus formas y prácticas de movilidad no solo están replegadas al espacio inmediato, es decir, el departamento o el barrio como antaño, más bien sus prácticas espaciales están ancladas a las múltiples escalas territoriales (centro, pericentro, periferia e incluso otra ciudad del país) y esferas que conforman su vida cotidiana. Algunos optan por espacios de proximidad y otros buscan la apertura hacia el resto del sistema metropolitano. A ello se suma que son habitantes insertos en un proceso de aumento del nivel de ingresos, y con ello mayores opciones de consumo de bienes y servicios y de elección residenciales" (Contreras, 2012: 24). En esta misma investigación se da cuenta de un amplio abanico de prácticas de movilidad por parte de los nuevos residentes en barrios en proceso de cambios socioeconómicos. Se reconocen prácticas de proximidad y contigüidad geográfica, en particular para el acceso al trabajo y para el uso cotidiano de comercio y servicios. Las formas de acceso incluyen caminatas, la utilización del transporte público (metro o buses) y del automóvil, aún para trayectos cortos. En algunos de los nuevos residentes se reconocen una combinación de estas prácticas de proximidad con otras de escala metropolitana, en particular, en relación con actividades que se despliegan durante los fines de semana (Contreras, 2012). Estas prácticas de movilidad están descriptas para los distintos grupos sociales de los barrios en proceso de gentrificación, lo que abre una interesante perspectiva de uso diferencial del territorio, aun con cierta coexistencia residencial.

La gran mayoría de los casos relevados de investigaciones que indagan en la importancia de la accesibilidad en las decisiones y prácticas de los grupos que comandan los procesos de gentrificación corresponden a ciudades europeas o de América del Norte. En ellos se enfatiza el atractivo que ofrecen las posibilidades de realizar recorridos peatonales, la facilidad para el uso de la bicicleta y para desarrollar trayectos cortos en transporte público, reduciendo notablemente la dependencia del automóvil particular. El patrón comparativo de los barrios gentrificados en las ciudades suizas analizados por Rérat y Lees (2010) da cuenta de la mayor importancia del transporte público y de los esquemas de movilidad sostenible, al tiempo que alertan sobre ciertos discursos que promueven supuestas bondades de la gentrificación sobre la base de esa sostenibilidad. Un patrón semejante es identificado por Barbonne (2008), para un barrio gentrificado de Montreal: distancias de desplazamiento menores al promedio de la ciudad, caminatas, uso de la bicicleta, utilización del transporte público, menos uso del automóvil.

Estos casos centrados en los grupos "gentrificadores" no deben hacernos perder de vista que el aprovechamiento de los esquemas de proximidad es particularmente crítico para los grupos sociales de ingresos medio-bajos que suelen localizarse en viviendas antiguas y habitualmente mal conservadas en los barrios del entorno al área central. Se trata de grupos débiles en términos de los distintos tipos de capital, pero con posibilidades de aprovechar el capital espacial fortalecido por la localización. De allí la relevancia de los desplazamientos que pueden implicar los procesos de gentrificación, y que se traducen, entre otras cosas, en pérdida o disminución de capital espacial.

En un contexto territorial bien diferente, como es el de la expansión urbana, también se ha hipotetizado sobre procesos de gentrificación, en tanto transformación del ámbito de residencia histórico de los grupos sociales de ingresos medio-bajos y bajos, a los que se superpone la llegada de los grupos de ingresos medio-altos y altos. En el caso particular de Buenos Aires, el proceso de suburbanización se desarrolló ampliamente a partir de la década de 1940 sobre la base de una política combinada de transporte y de acceso al suelo (Torres, 1993). Los ferrocarriles, orientadores de la expansión, gozaron de una tarifa barata, al tiempo que se promovieron loteos populares, lo que devino en un modelo de urbanización basado en el transporte público y protagonizado por los grupos de ingresos medios o medio-bajos, con una estructura de tipo tentacular. Este patrón tradicional se ve ampliamente trastrocado con el despliegue de un nuevo modelo de urbanización-movilidad a partir de la década de 1990, basado en el transporte individual, apoyado en la red de autopistas y en el desarrollo de nuevos productos inmobiliarios (las urbanizaciones cerradas), y protagonizado por sectores de ingresos medio-altos y altos. 
Álvarez Rivadulla traza una serie de paralelos significativos entre los grupos sociales residentes en las nuevas urbanizaciones de Montevideo y los que participan de procesos de gentrificación en diferentes partes del mundo, lo que habilitaría a considerar que "las comunidades cerradas en América Latina implican procesos de gentrificación" (2007: 60). Al mismo tiempo, da cuenta de la transformación de la periferia de muchas de las ciudades latinoamericanas en un "territorio en disputa" que puede implicar alzas de precios y desplazamientos de población residente. La autora propone prestar especial atención a la potencialidad de la investigación de estos fenómenos con mayor énfasis en los procesos y en las categorías sociales subyacentes que en las formas físicas.

Entendemos, no obstante, que estas formas físicas son calificadoras significativas de los procesos sociales y que dan cuenta del proceso de construcción social del territorio, con una secuencia de transformaciones físicas que evidencian las necesidades de reproducción social de los grupos sociales dominantes (Blanco, 2006). Se abre así una amplia discusión acerca de los rasgos comunes entre la periurbanización a través de las comunidades cerradas y los procesos de gentrificación. Estas modalidades de expansión urbana están estrechamente asociadas con las políticas neoliberales en América Latina, y suponen otros dos rasgos constitutivos de la gentrificación: "la llegada de agentes con mayor capacidad de pago que los usuarios establecidos en ese espacio o en áreas colindantes" y "cambios en las actividades y en el paisaje urbano controlados por los grupos que ingresan al territorio en cuestión" (Casgrain y Janoschka, 2013). Solo para dar una idea de los cambios en el paisaje urbano, son significativos los "nuevos objetos urbanos o artefactos de la globalización" identificados para el caso de la Región Metropolitana de Buenos Aires por Ciccolella (1999), ejemplificados con los centros comerciales y de servicios sobre autopistas, los colegios bilingües e internacionales, los inmuebles de oficinas en las periferias metropolitanas o los hoteles cinco estrellas. Estos objetos estaban reservados, hasta hace solo un par de décadas, a las áreas centrales de las grandes ciudades.
En este contexto territorial también juegan un rol relevante las nociones de accesibilidad, de conectividad y de proximidad, pero recalificadas en función de las particularidades de la expansión urbana: las ventajas están dadas por el acceso rápido a las redes de autopistas que facilitan la articulación metropolitana y la proximidad, y se miden en tiempo de uso del automóvil particular. Sostenemos para estos casos la denominación de "automóvil-intensivo" (Blanco, 2006) para describir al modelo de movilidad dominante, evitando así la más común denominación de automóvil dependiente (Newman \& Kenworthy, 2006), ya que se trata de una dependencia escogida. El modelo automóvil intensivo es el supuesto a partir del cual se construye ese fragmento del territorio $y$, al mismo tiempo, es el resultado de la construcción práctica de ese modelo de expansión urbana. Este resultado confirma la hipótesis de partida: es sumamente difícil el desplazamiento sin el automóvil. El capital espacial resignificado en la periferia está formado básicamente por la asociación autopistas -disponibilidad de automóvil- competencias para conducir. En los casos en que no se utiliza el propio vehículo, las urbanizaciones de cierta escala ofrecen servicios de combis o vans especiales propios, siempre de acceso restringido, y diferenciados del transporte público (Blanco, 2006, Álvarez Rivadulla, 2007), lo que no deja de ser apenas una variante del modelo automóvil intensivo.

Los procesos de periurbanización asociados con la expansión de las redes de autopistas metropolitanas reflejan muy bien esta relación entre urbanización y movilidad dominantes, dejando al menos dos cuestiones críticas en relación con los grupos sociales de bajos ingresos. Una primera cuestión es la conformación de una movilidad subordinada a los procesos de construcción social de ese espacio bajo el modelo automóvil intensivo. Un ejemplo de esta subordinación puede encontrarse en investigaciones sobre la movilidad de los trabajadores que se desempeñan en el interior de las urbanizaciones cerradas en la Región Metropolitana de Buenos Aires (Blanco et al., 2013). Una segunda cuestión crítica es la "apropiación de accesibilidad" (Blanco y San Cristóbal, 2012) manifiesta en el uso del frente de las autopistas y su área inmediata por parte de los grupos dominantes 
y en la relegación de los sectores populares a los espacios intersticiales más aislados de los principales ejes de circulación y con serias dificultades para el establecimiento de una red de transporte público que satisfaga las necesidades de movilidad. Aquí la pregunta clave también remite al conjunto de políticas urbanas que facilitan estos procesos de segregación, subordinación y exclusión, que pueden llevar a situaciones marcadas de inmovilidad relativa o insularidad (Le Breton, 2004) al tiempo que promueven una ciudad menos equitativa, más costosa en términos económicos y poco sostenible en el mediano plazo.

\section{Las consecuencias sobre la movilidad de los grupos desplazados}

El tercer eje propuesto pone el acento en las consecuencias que generan los procesos de gentrificación con desplazamiento de población sobre la movilidad de los grupos desplazados. Casgrain y Janoschka (2013) destacan como cuarto rasgo de los procesos de gentrificación: "el desplazamiento directo, o la presión indirecta para el desplazamiento, de grupos sociales de ingresos más bajos de los que entran" al área renovada. Los desplazados suelen ser obligados a dirigirse hacia lugares con menor accesibilidad, más lejanos a los empleos que ya tienen o a las áreas de empleo donde podrían encontrar trabajo, probablemente con menores alternativas de acceso a centros de servicios y desarmando las redes sociales tejidas en el barrio de residencia. Es decir, el desplazamiento implica una mudanza del "sistema residencial" que puede traducirse en pérdidas de accesibilidad o en un aumento de costos y distancias que impida continuar desarrollando sus actividades cotidianas.

Cobra relevancia, en consecuencia, la perspectiva de un planteo relacional que articula las capacidades de movilidad de distintos grupos sociales. Así es como lo expresan Rérat y Lees cuando sostienen que "si la gentrificación aparece como una estrategia para incrementar el capital espacial de algunos grupos de la clase media, puede tener el efecto opuesto sobre la población desplazada. Además de la pérdida de su barrio (y, por lo tanto, del capital social) por los procesos de gentrificación, los desplazamientos también pueden resultar en pérdida de capital espacial" (Rérat \& Lees, 2011: 129).

Barbonne (2008) señala, en su estudio sobre los barrios gentrificados de Montreal, la existencia de una importante atracción cotidiana hacia ellos de antiguos residentes que se movilizan para trabajar. Esto da cuenta de la ruptura o la extensión de las relaciones de proximidad previas entre residencia y trabajo, originadas en el desplazamiento de esos antiguos residentes hacia otros barrios. Rérat y Lees (2011) citan otros ejemplos de pérdida de capacidad para el viaje cotidiano hacia el trabajo en ciudades tan distintas como Shanghai y Ciudad del Cabo, ya sea por desplazamientos hacia la periferia o por exclusión e imposibilidad de acceso en una estrategia de mercado (Visser \& Kotze, 2008).

En América Latina son numerosos los estudios sobre exclusión y segregación vinculados con la movilidad, relacionados con las dificultades de acceso a suelo bien localizado y servido por redes de transporte. Conocidas son las consecuencias negativas de la periferización de los pobres urbanos, a partir de la segmentación desigual de los bienes y servicios urbanos, de la pérdida de activos locales y de la acumulación territorial de desventajas (Kaztman, 2001; Rodríguez y Sugranyes, 2004). Sin embargo, hay una carencia importante de estudios dirigidos directamente a recoger las nuevas condiciones de movilidad y acceso de la población desplazada a partir de procesos de gentrificación.

Este tercer eje, en consecuencia, se preocupa por la pérdida de capacidad de acceso de los sectores desplazados en el proceso de gentrificación y por la percepción y explicitación de estas cuestiones en los movimientos de resistencia. Algunas de las preguntas que orientan la indagación en este eje son: ¿Cómo se desplaza/descoloca/reconstruye el cuadro de relaciones y representaciones cotidianas a partir de los desplazamientos residenciales originados en procesos de gentrificación? ¿Qué lugares y sistemas de actividades anteriormente accesibles dejan de serlo? ¿Cómo se manifiesta la pérdida relativa de capital espacial en los grupos sociales desplazados? ¿Qué movimientos sociales y resistencias se generan en torno a estos procesos? ¿Son incluidas como demandas o, 
mejor aún, como derechos, las necesidades de movilidad?

\section{A modo de reflexión final}

El objetivo principal de este trabajo fue construir un marco conceptual que nos permita indagar en las relaciones entre los procesos de gentrificación, la infraestructura y los servicios de transporte y la movilidad cotidiana de la población. Mediante una revisión bibliográfica orientada a explorar los antecedentes y los casos en los que se vinculan conceptualmente o se analizan empíricamente estas relaciones entre gentrificación, transporte y movilidad, dimos cuenta de un entramado conceptual que abre las puertas para la constitución de una agenda de investigación.

A partir de esta explicitación conceptual hemos propuesto tres ejes de indagación relacionados con los efectos de las inversiones en materia de transporte como posible dispositivo gentrificador (Sequera, 2013), con la atracción diferencial de la accesibilidad en el despliegue de procesos de gentrificación y con los efectos que los desplazamientos de grupos sociales más vulnerables tienen sobre la movilidad cotidiana y el acceso a recursos urbanos. En cada uno de ellos hemos planteado preguntas generales orientadoras de las futuras etapas de indagación que estarán ancladas, necesariamente, en el desarrollo de los estudios de caso.

Entendemos que la incorporación de los cambios socioterritoriales vinculados con el transporte y la movilidad puede traer nuevas dimensiones en el análisis de los procesos de gentrificación en, al menos, dos cuestiones principales.

En primer lugar, en las ciudades de América latina hay serios problemas de transporte y movilidad cotidiana que requieren ser abordados, al tiempo que hay una rica tradición en estudios sobre la exclusión y la desigualdad social vinculada con estos problemas. Una mirada combinada con el estudio de los procesos de gentrificación puede aportar nuevos elementos de comprensión de la realidad urbana latinoamericana.

En segundo lugar, las problemáticas del campo gentrificación-transporte-movilidad interpelan las políticas públicas urbanas. Por intención deliberada o por omisión es muy posible que estas políticas estimulen mecanismos de gentrificación o no den respuestas a los derechos de los grupos sociales afectados por estos procesos. En definitiva, es una manera más de Ilamar la atención sobre la necesidad de implementar políticas públicas integrales que enfrenten las desigualdades intrínsecas de las regulaciones territoriales de mercado.

\section{Referencias bibliográficas}

ÁlVAREZ RIVADULLA, M.J. Golden ghettos: Gated communities and class residential segregation in Montevideo, Uruguay. Environment and Planning A, 2007, $\mathrm{N}^{\circ} 39$, p. 47-63.

ASCHER, F. Les sens du mouvement. Paris: Belin, 2004.

AVELLANEDA, P. y LAZO, A. Aproximación a la movilidad cotidiana en la periferia pobre de dos ciudades latinoamericanas. Los casos de Lima y Santiago de Chile. Revista Transporte y Territorio, 2011, No 4, p. 47-58.

BARBONNE, R. Gentrification, nouvel urbanisme et évolution de la mobilité quotidienne: Vers un développement plus durable? Le cas du Plateau Mont-Royal (1998-2003). Recherches sociographiques, 2008, Vol. 3, Nº 49, p. 423-445.

BAVOUX, J.; BEAUCIRE, F.; CHAPELON, L. \& ZEMBRI, P. Géographie des Transports. Paris: Armand Colin, 2005.

BLANCO, J. De la noción de impacto a la de procesos asociados. Reflexiones a partir de la relación autopistas-urbanización en la Región Metropolitana de Buenos Aires. Mundo Urbano, 2006, № 28. Disponible en Internet: http://www.mundourbano.unq.edu.ar

BLANCO, J. Notas sobre las relaciones transporte - territorio: Implicancias para la planificación y una propuesta de agenda. Revista Transporte y Territorio, 2010, № 3. p. 172-190.

BLANCO, J. y SAN CRISTÓBAL, D. Reestructuración de la red de autopistas y 
metropolización en Buenos Aires. Revista Iberoamericana de Urbanismo, 2012, N 8, p. 73-88.

BLANCO, J.; BOSOER, L.; GAMBA BARY, F. y SAN CRISTÓBAL, D. Movilidad cotidiana y trabajo. El caso de los trabajadores en urbanizaciones cerradas de la Región Metropolitana de Buenos Aires. La Plata: Tercer Concurso Bialet Massé. 2013.

CASGRAIN, A. y JANOSCHKA, M. Gentrificación y resistencia en las ciudades latinoamericanas. El ejemplo de Santiago de Chile. Andamios. Revista de investigación Social, 2013, N²2, p. 19-44.

CEBOLLADA, À. Aproximación a los procesos de exclusión social a partir de la relación entre el territorio y la movilidad cotidiana. Documents d'Anàlisi Geogràfica, 2006, $\mathrm{N}^{\circ} 48$, p. 105-121.

CEBOLLADA, A. Mobility and labour market exclusión in the Barcelona Metropolitan Region. Journal of Transport Geography, 2009, No 17 , p. 226-233.

CEPAL. Provisión de infraestructura de transporte en América Latina: experiencia reciente y problemas observados. Santiago de Chile: CEPAL, 2005.

CICCOLELLA, P. Globalización y dualización en la Región Metropolitana de Buenos Aires: Grandes inversiones y reestructuración socioterritorial en los años noventa. EURE, 1999, Vol. 76, N²5, p. 5-27.

CONTRERAS, Y. Cambios socio-espaciales en el centro de Santiago de Chile: formas de anclarse y prácticas urbanas de los nuevos habitantes. Santiago de Chile: Tesis de Doctorado en Arquitectura y Estudios Urbanos, Pontificia Universidad Católica de Chile, 2012.

CRESSWELL, T. On the move. Mobility in the Modern Western World. Nueva YorkLondres: Routledge, 2006.

CUENYA, B. y CORRAL, M. Empresarialismo, economía del suelo y grandes proyectos urbanos: el modelo de Puerto Madero. EURE, 2011, Vol. 111, N³7, p. 25-45.
DÁVILA, J. y DASTE, D. Pobreza, participación y Metrocable. Estudio del caso de Medellín. Boletin CF+S, 2011, No 54, p. 121 131.

DESFOR, G.; LAIDLEY, J.; STEVENS, Q. \& SCHUBERT, D. Transforming Urban Waterfronts: Fixity and Flow. Nueva York: Routledge, 2011.

DÍAZ OLIVERA, L.; PLAT, D. y POCHET, P. Caminar y nada más. Movilidad diaria y pobreza en las ciudades subsaharianas. Scripta Nova. Revista Electrónica de Geografía y Ciencias Sociales, 2008, No 261. Disponible en Internet: http://www.ub.edu/geocrit/novaig.htm

DUREAU, F. Les mobilités à géométrie variable des habitants de Bogota. Espace, populations, sociétés, 1999, N², p. 329-344.

FIGUEROA, O. Transporte urbano y globalización. Políticas y efectos en América Latina. EURE, 2011, Vol. 94, No 31, p. 41-53.

GRUBE-CAVERS, A. \& PATTERSON, Z. Urban Rapid Rail Transit and Gentrification in Canadian Urban Centres - A Survival Analysis Approach. Montreal: CIRRELT, 2013.

GUTIÉRREZ, A. Movilidad o inmovilidad, ¿qué es la movilidad? Aprendiendo a delimitar los deseos. Buenos Aires: ALATPU, 2009.

JANOSCHKA, M.; SEQUERA, J. \& SALINAS, L. Gentrification in Spain and Latin America. A critical dialogue. International Journal of Urban and Regional Research, 2013, Vol. 38, No 4, p. 1234-1265.

JOUFFE, Y. Las clases socio-territoriales entre movilidad metropolitana y repliegue barrial. ¿Tienen los pobladores pobres una movilidad de clase? Revista Transporte y Territorio, 2011, N4, p. 84-117.

KAUFMANN, V.; BERGMAN, M. \& JOYE, D. Motility: Mobility as Capital. International Journal of Urban and Regional Research, 2004, N²8, p. 745-756.

KAZTMAN, R. Seducidos y abandonados: el aislamiento social de los pobres. Revista de la CEPAL, 2001, N 75, p. 171-189. 
LE BRETON, E. Mobilité, exclusión et marginalité. In: ALLEMAND, S.; ASCHER, F. \& LÉVY, J. (directores). Les sens du mouvement. Paris: Belin. 2004, p. 117-123.

LEIBLER, L. y BRAND, P. Movilidad e inclusión social: la experiencia desde la periferia de Medellín y el primer Metrocable. Bulletin de I'Institut Français d'Études Andine, 2012, Vol. 3, N 41, p. 363-387.

LEVY, J. Os novos espaços da mobilidade. GEOgraphia, 2001, № 6, p. 7-22.

MASSEY, D. Um sentido global do lugar. In: ARANTES, A. (organizador). O Espaço da Diferença. Campinas: Papirus, 2000, p. 177 186.

MIRALLES-GUASCH, C. Ciudad y transporte. Barcelona: Ariel. 2002.

NEWMAN, P. \& KENWORTHY, J. Urban Design to Reduce Automobile Dependence. Opolis, 2006, Vol. 1, No 2, p. 35-52.

OFFNER, J.M. Les "effets structurants" du transport: mythe politique, mystification scientiphique. L'Espace Geographique, 1993, $\mathrm{N}^{\circ} 3$, p. 233-242.

ORFEUIL J.P. Transports pauvretés exclusions. Paris: Éditions de l'Aube, 2003.

POLLACK, S.; BLUESTONE, B. \& BILLINGHAM, Ch. Maintaining Diversity in America's Transit-Rich Neighborhoods: Tools for Equitable Neighborhood Change. Boston: Dukakis Center Northeastern University, 2010.
RERAT, P. \& LEES, L. Spatial capital, gentrification and mobility: evidence from Swiss core cities. Transactions of the Institute of British Geographers, 2011, №36, p. 126-142.

RODRÍGUEZ, D. \& MOJICA, C. Land Value Impacts of Bus Rapid Transit. The case of Bogotá's Transmilenio. Land Lines, 2008, N ${ }^{\circ}$ 4. Disponible en internet: http://equinoxcenter.org

RODRÍGuEZ, A. y SUGRANYES, A. El problema de vivienda de los "con techo". EURE, 2004, Vol. 91, №30, p. 53-65.

SANTOS, M. A Natureza do Espaço. Técnica e Tempo, Razão e Emoção. San Paulo: Hucitec, 1996.

SEQUERA, J. Nuevas clases medias, producción cultural y gestión del espacio público. El caso de Lavapiés en el centro histórico de Madrid. Madrid: UCM, 2013.

TORRES, H. El mapa social de Buenos Aires, 1940-1990. Buenos Aires: FADU-UBA, 1993.

URRY, J. Mobilities. Cambridge: Polity, 2007.

VERLANDIA, D. El impacto del sistema de Transporte Rápido de Buses (Metrobús) en los precios del suelo en la Ciudad de México. Cambridge (Massachusetts): Lincoln Institute of Land Policy, 2013.

VISSER, G. \& KOTZE, N. The State and New-build Gentrification in Central Cape Town, South Africa. Urban Studies, 2008, N ${ }^{\circ}$ 45, Vol. 12, p. 255-259. 
